\title{
Social Scoring: Evaluation qualifizierender Beschäftigung
}

\author{
Stefanie Ernst \\ Uwe Hartwig \\ Felizitas Pokora
}

Der Erfolg arbeitsmarktpolitischer Beschäftigungsmaßnahmen wird unter dem neuen, autoritär-aktivierenden Paradigma vorwiegend an der Integration in Beschäftigung gemessen. Dieses Ziel geht an den Voraussetzungen und Möglichkeiten besonders arbeitsmarktferner Arbeitsloser vorbei. Zugleich bedeutet es, dass Maßnahmen, die realistischere Vorstufen zur Arbeitsmarktbeteiligung wie Empowerment und soziale Teilhabe verbessern, entlang vorherrschender Erfolgskriterien nur unzureichend evaluiert werden können. Mit der Social Balanced Scorecard erhalten die Träger der Maßnahmen ein Instrument, erweiterte Zielsetzungen der Arbeitsmarktpolitik systematisch zu verfolgen und ihren Erfolg zu überprüfen.

\section{Einleitung}

Die Arbeitsmarktpolitik vollzog in den letzten Jahren einen Paradigmenwechsel, indem sie die Eigenverantwortung des Einzelnen stärker einfordert und eine Philosophie des „Förderns und Forderns“ verfolgt, ungeachtet der Tatsache, dass Arbeitslosigkeit in den meisten Fällen nicht selbstverschuldet ist. Dieser Paradigmenwechsel reflektiert zum einen zu wenig die Entwicklungen am Arbeitsmarkt. Zum anderen überfordert die Eigenverantwortung viele langzeitarbeitslose wie auch leistungsgeminderte Menschen, während die konkreten Maßnahmeangebote sie unterfordern.

Dass bei einer größeren Gruppe der Betroffenen das Kriterium der direkten Vermittlung in den ersten Arbeitsmarkt nicht als alleiniges Erfolgskriterium greift, zeigt die zwanzigjährige Erfahrung von Qualifizierungs- und Beschäftigungsunternehmen. Zudem verlieren diejenigen, die nach ihrem Arbeitsplatzverlust nicht schnell eine Anschlussbeschäftigung finden, oft ihre beruflichen Perspektiven, langfristig gar ihre sozialen Bezüge und gesellschaftlichen Teilhabemöglichkeiten. Deshalb ist bei der Umsetzung der seit Januar 2005 begonnenen arbeitsmarktpolitischen Maßnahmen das Empowerment von Langzeitarbeitslosen als ein zentraler Aspekt der Betreuung bzw. Begleitung und Integrationsförderung besonders zu beachten. Dabei lassen sich die Teilhabechancen Betroffener durch ein sogenanntes „Social Scoring"1 ermitteln, sodass die gegenwärtig bestehenden Instrumente zur Evaluation arbeitsmarkt- politischer Maßnahmen unter dem Begriff Empowerment um Dimensionen sozialer Teilhabe ergänzt werden können. „Social Scoring "kann als Teil einer erweiterten Balanced Score Card, einer „Social Balanced Scorecard" dazu beitragen, die Wirkungen von Qualifizierungs- und Beschäftigungsmaßnahmen aufzuzeigen.

Im Folgenden wird „Social Scoring“ als ein innovatives Messinstrument zur Erfassung der Effizienz qualifizierender Beschäftigung in Beschäftigungs- und Qualifizierungsunternehmen in Form einer Zwischenbilanz dargestellt. Sie basiert auf ersten Ergebnissen eines explorativen Begleitforschungsprojektes, dessen Erhebung und Auswertung von Januar bis September 2007 erfolgt ist. ${ }^{2}$ Die Entwicklung der grundlegenden Fragestellung, wie effizient qualifizierende Beschäftigung auf der Grundlage der aktuellen Arbeitsmarktpolitik ist, basiert sowohl auf den praxisnahen Erfahrungen in einer Frankfurter und einer Hamburger Beschäftigungs- und Qualifizierungsgesellschaft als auch auf der wissenschaftlichen Reflexion der heute gültigen Reformansätze.

Zunächst wird die aktuelle Arbeitsmarktpolitik mit Blick auf die Auswirkungen für die Betroffenen und Beteiligten (Arbeitslose, Institutionen und Sozialunternehmen) beschrieben (Abschnitt 2). Die Begriffe Empowerment und soziale Teilhabe werden sodann theorie- und praxisorientiert in den Kontext von Social Scoring eingefügt (Abschnitt 3) und ihre Umsetzung in der explorativen Studie dargestellt (Abschnitt 4). Erste Ergebnisse der Studie deuten darauf hin, dass qualifizierende Beschäftigung in Sozialunternehmen bisher nicht in ausreichendem Maß die Kompetenzen der Selbstorganisation und -vermarktung vermittelt, die auch von Arbeitslosen vermehrt gefordert werden (Abschnitt 5.1). Mit einer kurzen Zusammenfassung der Stärken des Messinstruments und einem Ausblick auf die weitere Forschung schließt der Beitrag (Abschnitt 5.2).

\footnotetext{
1 Der Begriff "Scoring" verweist auf eine Herangehensweise, die auf die Ermittlung von Kennzahlen zielt; hier zum Themenfeld personale Kompetenzen.

2 Der Beitrag basiert auf dem Kooperationsprojekt "Innovation in der Sozialwirtschaft", das Teil des EQUAL-Projekts der EU-Gemeinschaftsinitiative "Wandel - Innovation - Botschaft" ist.
}

Stefanie Ernst, Prof. Dr., Juniorprofessur Arbeit, Organisation, Geschlechterverhältnisse, Universität Hamburg, Department Wirtschaft und Politik. Arbeitsschwerpunkte: Arbeits- und Organisationssoziologie, Geschlechterforschung, Soziologische Gesellschaftstheorie, Zivilisationstheorie, Methoden der Sozialforschung

e-mail: Stefanie.Ernst@wiso.uni-hamburg.de Uwe Hartwig, Dipl.Ökonom, Werkstatt Frankfurt. Arbeitsschwerpunkte: Management sozialer Organisationen, Reporting und Controlling, Entwicklungspolitik. e-mail: uwe.hartwig@werkstatt-frankfurt.de Felizitas Pokora, Dr., Netz NRW Verbund für Ökologie und soziales Wirtschaften e.V, Köln. Arbeitsschwerpunkte: Erwachsenenbildung, Qualitäts-/Organisations- und Personalentwicklung, Arbeitsforschung, Work Skills, Genderforschung. e-mail: felizitas.pokora@netz-nrw.de 


\section{Paradoxe Anreize und Zwänge für Arbeitsuchende}

Mit dem Übergang vom nationalstaatlichen Modell des „rheinischen Kapitalismus“ zum Modell eines globalisierten, neoliberal-marktorientierten Kapitalismus (Bereitstellung optimaler Wettbewerbsbedingungen durch Steuersenkung, Entbürokratisierung und Entstaatlichung) haben sich vor allem die Arbeitsverhältnisse als Basis sozialer Integration geändert. Prekäre und atypische Beschäftigungsverhältnisse nehmen zu, das Normalarbeitsverhältnis erodiert, Armut und soziale Polarisierung wachsen. Gering qualifizierte Erwachsene, ältere Arbeitnehmer, Jugendliche ohne Schulabschluss sowie Menschen mit geringem kulturellen Kapital geraten durch diese Entwicklung vermehrt in Langzeitarbeitlosigkeit. Durch die Abkoppelung von der Teilhabe am Arbeitsmarkt entfernen sie sich von den Anforderungen auf dem ersten Arbeitsmarkt, das heißt: Sind diese Menschen mehrere Monate ohne sozialversicherungspflichtige Beschäftigung, wachsen für sie die Risiken einer dauerhaften Erwerbslosigkeit im Zuge ihres wachsenden Produktivitätsverlustes (Ludwig-Mayerhofer 2005, S. 211).

Gegenwärtig konzentriert sich die Arbeitsmarktpolitik in Umsetzung der HartzGesetze darauf, die Anreize zur Arbeitsaufnahme zu erhöhen, die Vermittlungsbemühungen zu verstärken und nicht-aktive Erwerbslose nicht nur zu ermitteln, sondern auch empfindlich zu sanktionieren. Die dazu bundesweit gegründeten Arbeitsgemeinschaften (ARGEn) und kommunalen Institutionen betreiben seitdem auf regional höchst unterschiedliche Weise Beschäftigungsförderung. Zwar wurden bis heute Mitteleinsparungen realisiert. So führten unter anderem statistische Bereinigungen und eine gute Konjunkturlage zu einer deutlichen und nachhaltigen Entspannung auf dem Arbeitsmarkt. Aber auf der anderen Seite bewirkten die Hartz-Gesetze eine zunehmende Verarmung und Arbeitsmarktferne. Nicht unwesentlich war daran die Ausgestaltung von Arbeitsgelegenheiten (AGHs) beteiligt.

Die Grundsicherung für Arbeitsuchende wird seit Januar 2005 im Rahmen des vierten Gesetzes für moderne Dienstleitungen am Arbeitsmarkt (Hartz-IV) neu geregelt, d.h. Arbeitslosen- (SGB III) und
Sozialhilfe für erwerbsfähige Sozialhilfeempfänger (BSHG-Bundessozialhilfegesetz) wurden zum Arbeitslosengeld II (ALG II) zusammengelegt (Renn/Schoch 2005). ${ }^{3}$ Für viele erwachsene langzeitarbeitslose ALG-II-Empfänger mit schlechten Bewerbungserfahrungen bleibt das Instrument der Arbeitsgelegenheiten nach $\S$ 16 (3) SGB II die einzige Maßnahme aktiver Arbeitsmarktpolitik. ${ }^{4}$ Die im Gesetz vorgesehenen Maßnahmen, wie die Förderung der Betreuung von Kindern und pflegebedürftigen Angehörigen ( $\$ 16$ (2) SGB II) und weitere fördernde Maßnahmen finden bisher kaum Anwendung. Mit dem Instrument der Arbeitsgelegenheiten konnte die Integration in den Arbeitsmarkt erwartungsgemäß nicht erhöht werden (Kettner/Rebien 2007).

Sogar die immer seltener eingesetzten Arbeitsbeschaffungsmaßnahmen (ABM) bieten oft qualifizierende Beschäftigung, die näher an den Bedingungen des ersten Arbeitsmarkts liegt, wie die Hamburger Studie „Evaluation der Maßnahmen zur Umsetzung der Vorschläge der HartzKommission" (Compass et al. 2006) zeigt. Während das Ziel, Beschäftigungsfähigkeit zu erhalten bzw. wiederherzustellen, durch $\mathrm{ABM}$ weitgehend erreicht wurde, bleibt ihr arbeitsmarktpolitischer Einsatz marginal (Compass et al. 2006, S. 136f.). Allerdings kann Arbeitsmarktintegration auch hier "nur bei einem intakten regionalen Arbeitsmarkt" (ebd., S. 141) gelingen.

Die befristeten Arbeitsgelegenheiten sind dagegen für leistungsgeminderte Erwerbslose gedacht, die in der Sprache der Bundesagentur für Arbeit (BA) als sogenannte ,marktferne“ Kunden geführt werden. Dennoch sollen - neben der Überprüfung der Arbeitsbereitschaft - schwer vermittelbare Personen durch Arbeitsgelegenheiten wieder an den Arbeitsmarkt herangeführt werden. Diese Aktivierungsstrategie funktioniert jedoch kaum, wenn die Handlungsautonomie der Träger stark eingeschränkt wird, indem die Jobs nur zusätzlich, ohne Konditionen einer regulären Beschäftigung bzw. ohne marktnahe Arbeitskontexte, ausgelegt sein dürfen. Ebenso kann das Ziel einer Erhöhung der Eigenverantwortlichkeit nicht erreicht werden, wenn die Beschäftigten z. B. für ihre berufliche Weiterbildung eigenverantwortlich Vorsorge treffen sollen und von ihnen die Kompetenz gefordert wird, selbstverantwortlich die marktrelevanten Informationen zu beschaffen und zu verarbeiten.
Demgegenüber lassen sich auch im gegebenen institutionellen Kontext, so unsere These, mit einer intensiven Begleitung, mit gezielter Kompetenzerweiterung und mit Ansätzen eines Empowerments durchaus Erfolge bezüglich einer Integration in den Arbeitsmarkt und besonders einer verbesserten gesellschaftlichen Teilhabe erzielen.

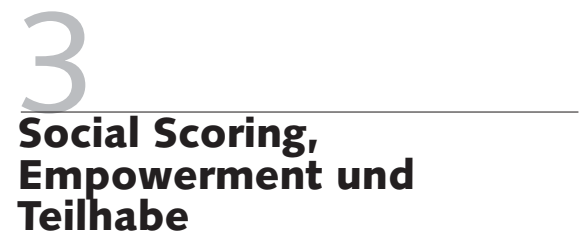

Vor diesem Hintergrund stellt sich die Frage, wie die Umsetzung der neuen Arbeitsmarktpolitik evaluiert und Qualifizierungsund Beschäftigungsmaßnahmen auf ihren Beitrag zur Integration untersucht werden können. Wie können dabei Träger von Qualifizierungs- und Beschäftigungsmaßnahmen ihre Angebote optimal ausrichten und steuern? Die Integrationsquote in den allgemeinen Arbeitsmarkt kann nicht mehr alleiniges Kriterium für den Erfolg von arbeitsmarktpolitischen Dienstleistungen sein, da dieser für einen großen Teil langzeitarbeitsloser Menschen nicht erreichbar ist. Für die Evaluation der Wirkungen qualifizierender arbeitsmarktpolitischer Maßnahmen ist es mehr noch erforderlich, zusätzlich die Erfolge bei sozialer Integration, verbesserter Gesundheit und sozialer Kompetenzentwicklung zu dokumentieren. Die vorhandenen Instrumente zur Evaluation von Maßnahmen müssen hier weiter entwickelt werden, da sie sich bislang nur auf die Integration in sozialversicherungs-

3 Dabei sind auch die Zuständigkeiten für bestimmte Leistungen der Erwerbslosen, die in den Zuständigkeitsbereich des SGB II fallen, zum einen auf die Bundesagentur für Arbeit und zum anderen auf die Kommunen (kreisfreie Städte und Landkreise) verteilt worden. Um ihre Zusammenarbeit zu verbessern, haben sich in vielen Kommunen die eingangs erwähnten Arbeitsgemeinschaften (ARGEn) gebildet.

4 „Für erwerbsfähige Hilfebedürftige, die keine $\mathrm{Ar}$ beit finden können, sollen Arbeitsgelegenheiten geschaffen werden. Werden Gelegenheiten für im öffentlichen Interesse liegende, zusätzliche Arbeiten nicht nach Absatz 1 als Arbeitsbeschaffungsmaßnahmen gefördert, ist dem erwerbsfähigen Hilfebedürftigen zuzüglich zum Arbeitslosengeld II eine angemessene Entschädigung für Mehraufwendungen zu zahlen; diese Arbeiten begründen kein Arbeitsverhältnis im Sinne des Arbeitsrechts (...). “ (§ 16 (3) SGB II). 
pflichtige Beschäftigungsverhältnisse konzentrieren und wenig ausdifferenziert sind (vgl. Eichhorst/ Zimmermann 2007, S. 4, 7; Becker/Moses 2004, S. 34ff.). Mehr noch stellt Baetghe-Kinsky $(2006,2007)$ angesichts der ersten Zwischenberichte zur Hartz-Gesetzgebung fest, dass für die komplexen und individuellen Problemlagen der Arbeitsuchenden „kein Tiefenprofiling gemäß etablierten Standards“, sondern nur eine "Grobsortierung nach Kundengruppen“" (Baetghe-Kinsky 2006, S. 4) stattfinde. Die bislang vorliegenden Evaluationsberichte ließen mithin wenig belastbare und nachvollziehbare Handlungsempfehlungen erkennen. Um mehr zu ermitteln als wenig aussagekräftige Integrationsquoten allein, müssten die eingesetzten ökonometrischen Methoden verfeinert werden. Was genau Beschäftigungsfähigkeit bzw. Employability oder Integration ausmacht, werde in einer rein datengetriebenen Wirkungsforschung übersehen. Nachhaltige Wirkungen auf die Erwerbsbiografie und „adressatenbezogene Erfolgskriterien“ (ebd., S. 9), wie z. B. Stabilisierung des Erwerbslosen, „Korrektur des missmatch“ (Penz 2007, S. 3), sollten daher vermehrt als kontext- und situationsspezifische Indikatoren aufgegriffen werden. Deck/Kruppe (2003) und die Compass-Studie (Compass et al. 2006) verweisen hier explizit auf die Vieldeutigkeit und Komplexität des dynamisch zu betrachtenden Begriffs der Beschäftigungsfähigkeit. Direkte Kausalzusammenhänge zwischen Förderung und Erfolg oder Misserfolg von arbeitsmarktpolitischen Maßnahmen ließen sich weniger verifizieren als vielmehr nur vermuten (Deck/Kruppe 2003, S. 7, 12, 73f.), sodass der Blick auf die Veränderungsprozesse und Heterogenität der Zielgruppen zu richten sei.

Daher ist unser Anliegen anders angelegt als etwa in der Hamburger Evaluationsstudie (Compass et al. 2006). Während es dort darum geht, zu überprüfen, ob und wie die Ziele der Maßnahmen u.a. in Form von Brutto- und Nettointegrationseffekten erreicht wurden, wird hier die praktische Umsetzung fokussiert und die Zielstellung der Arbeitsgelegenheiten weiter ausdifferenziert. Sowohl die Förderung der Beschäftigungsfähigkeit und gesellschaftlichen Teilhabemöglichkeiten als auch die Stärkung der Fähigkeiten, eigene Ressourcen zu aktivieren (Empowerment) stehen dabei im Vordergrund. Die Rolle von Sozialunternehmen stellt in diesem Kontext ein Spezifikum und ein bislang „untererforschtes Phänomen“ (Koch/Kupka 2007, S. 22) dar. Zum einen müssen sich immer häufiger Erwerbstätige um- und neu orientieren und während des gesamten Erwerbslebens Lerngelegenheiten nutzen, um ihre Beschäftigungsfähigkeit zu erhalten und zu steigern. Im Arbeitsprozess steigt der Anteil von nicht routinisierbaren und variierenden Aufgabenstellungen, und die Anforderungen an die Arbeitnehmer werden in Zukunft vor allem nach modernen Schlüsselkompetenzen, ${ }^{5}$ wie beispielsweise Entscheidungs-, Reflexions- und Teamfähigkeit sowie Kreativität u.a.m. (vgl. Tractenberg et.al. 2002), ausgerichtet. Diese gewachsenen Anforderungen des ersten Arbeitsmarktes verweisen zudem auf eine zunehmende Subjektivierung des Arbeitshandelns und die Einbeziehung personaler Kompetenzen der Beschäftigten, die nicht durch formelle Qualifikationen abgedeckt werden und schwerer überprüfbar sind als reines Fachwissen. Zum anderen zeigt sich, dass für Personen, die in den ersten Arbeitsmarkt vermittelt werden sollen bzw. deren Beschäftigungsfähigkeit (wieder) hergestellt werden soll, die Vermittlung dieser Kompetenzen von hoher Bedeutung ist.

Bei der Zielgruppe von qualifizierenden Beschäftigungsmaßnahmen ist daher vom Ansatz des Empowerments und von einem auszubauenden Niveau der personalen Kompetenzen auszugehen. Social Scoring will in diesem Kontext messen und dokumentieren, ob und inwiefern die Kompetenzen gering qualifizierter Erwerbsloser im Rahmen eines weitergehenden Integrationsansatzes verbessert werden. Empowerment meint dabei „den Prozess, innerhalb dessen Menschen sich ermutigt fühlen, ihre eigenen Angelegenheiten in die Hand zu nehmen, ihre eigenen Kräfte und Kompetenzen zu entdecken und ernst zu nehmen und den Wert selbst erarbeiteter Lösungen schätzen zu lernen“" (Kreft/Mielenz 2005, S.234.). ${ }^{6}$

Auf der individuellen Ebene der Betroffenen geht es damit um die Aneignung von Handlungsfähigkeit, Energie und Gestaltungsvermögen, sodass die Betroffenen zu Akteuren werden. Professionell Helfende unterstützen auf der institutionellen Ebene die Bereitstellung von Ressourcen für Empowerment-Prozesse, was gerade im Hinblick auf die gewachsenen Anforderungen des ersten Arbeitsmarktes und potenzieller Arbeitgeber von besonderer Bedeutung ist. Ansätze in der sozialen Arbeit soll- ten demnach auch immer die ganze Person und ihre Autonomie stärken, um adäquat auf diesen Arbeitsmarkt vorzubereiten.

Empowerment setzt darüber hinaus auch darauf, Vereinzelung zu überwinden und zu bewirken, dass der Einzelne lernt, sich wieder in Gemeinschaften einzufügen, sich aktiv Informationen und Dienstleistungen zu verschaffen und das eigene Recht auf Teilhabe und Mitwirkung einzufordern. Damit wird die bisherige Konzentration auf Defizite zugunsten einer Sichtweise verworfen, die den Klienten als kompetenten Akteur sieht (Herriger 1997). Aus diesem Grund ist neben der gängigen Fremd- auch die Selbsteinschätzung der Teilnehmenden relevant.

Als weiterer Bezugspunkt des Social Scoring lässt sich gesellschaftliche Teilhabe ausmachen. Sie wird an den Handlungsspielräumen gemessen, die eine individuell gewünschte oder gesellschaftlich übliche Lebensweise ermöglichen. In Anlehnung an Kronauer (2001) lassen sich vier Bereiche von Teilhabe unterscheiden: gesellschaftliche Arbeit, soziale Nahbeziehungen, Rechte und Kultur. Gefährdet (prekär) wird Teilhabe dann, wenn die äußeren sozialen Anforderungen nicht mehr mit den Möglichkeiten zu ihrer Realisierung übereinstimmen. Diese Gefährdung kann bei anhaltendem Sozialabbau nicht nur in Ausgrenzung umschlagen, sondern vielmehr langfristig „Stoff für eine neue, soziale Frage“ liefern, die „von derselben Tragweite und Zentralität wie jene ist, die der Pauperismus in der ersten Hälfte des 19. Jahr-

5 Schlüsselkompetenzen umfassen „Kenntnisse und Fähigkeiten, welche nicht unmittelbaren und begrenzten Bezug zu bestimmten (...) Tätigkeiten erbringen" (Mertens 1974) und angesichts des schnell veraltenden Wissens überdauern, um das Leben in einer modernen Gesellschaft zu ermöglichen. Dabei reichen die Anforderungen an Arbeitskräfte heute bis in die "Kernbereiche der Persönlichkeit“" hinein (Knauf 2001, S. 46). Mit den Begriffen Sozial-, Methoden-, Selbst- und Fachkompetenz wird in der gegenwärtigen Debatte eine Weiterentwicklung des Diskurses markiert, die wir für Empowerment in Lernkompetenzen und -bereitschaft, Organisationskompetenz, sozialkommunikative und Sprachkompetenz sowie Leistungs- und Fachkompetenz ausdifferenziert haben.

6 Diese umfassende Definition von Empowerment in sozialer Arbeit trifft nicht mehr das aktuelle Verständnis von Beschäftigungs- und Qualifizierungsgesellschaften, die im Rahmen des „Förderns und Forderns" weit weniger sozialpädagogische Betreuung und Unterstützung durch professionelle Helfer leisten sollen. 
hunderts zum allgemeinen Erstaunen der Zeitgenossen auslöste" (Castel 2000, S. 357; vgl. Bartelheimer 2004).

\section{Die explorative Studie}

\subsection{SOZIALUNTERNEHMEN}

Die Studie wurde in zwei großen Sozialunternehmen in Hamburg und Frankfurt durchgeführt. Ihrem Charakter nach sollen sie das Gemeinwohl fördern, ohne Eigeninteressen $\mathrm{zu}$ verfolgen. Sie handeln aber gleichwohl nach Effizienzgrundsätzen. Bei ihren Aktivitäten steht nicht die Gewinnmaximierung im Zentrum, sondern immer der Mensch mit seinen Bedürfnissen und seinem Recht auf Würde. Auf diese Aufgabenstellung ist die Social Balanced Scorecard zugeschnitten.

Als bundesweit größte kommunale Anbieter offerieren die zwei Sozialbetriebe Beschäftigungs- und Qualifizierungsmaßnahmen, um die Fähigkeit zur Selbsthilfe zu stärken. Dabei sollen langzeitarbeitslose Menschen auf den allgemeinen Arbeitsmarkt vorbereitet und Beschäftigung hemmende Faktoren abgebaut werden (Osenberg 1995, S. 58ff.). Ihre Konzepte unterscheiden sich jedoch nach Arbeitsmarktnähe und Dauer der qualifizierenden Beschäftigung.

Die Hamburger Arbeit (HAB 2005) bietet mit ihrer 2002 eingeführten Vorbereitungs- und Integrationsphase sowohl Qualifizierung an als auch die Möglichkeit, Förderwilligkeit zu überprüfen. Durch eine Integrationsphase (IP) sollen sich erwerbslose Personen über 25 Jahre zum einen wieder an einen geregelten Arbeitstag gewöhnen und zum anderen soweit fachlich qualifizieren, dass sie erneut in den regulären Arbeitsmarkt eingegliedert werden können. Die Regelförderdauer für Arbeitsgelegenheiten (AGH) beträgt maximal zehn Monate. Davor liegt eine Vorbereitungsphase (VP), in der die Fähigkeiten der Teilnehmenden für angebotene Tätigkeiten ermittelt werden sollen.

Die Werkstatt Frankfurt organisiert zwar ebenfalls Arbeitsgelegenheiten, überwiegend bei Kooperationspartnern, setzt mit ihrem bundesweit beachteten „Frankfurter Weg zum Berufsabschluss" jedoch stärker auf modulare berufliche Qualifizierung in eigenen Betrieben, weil marktnahe

\section{Abb. 1: Social Balanced Scorecard für Sozialunternehmen}

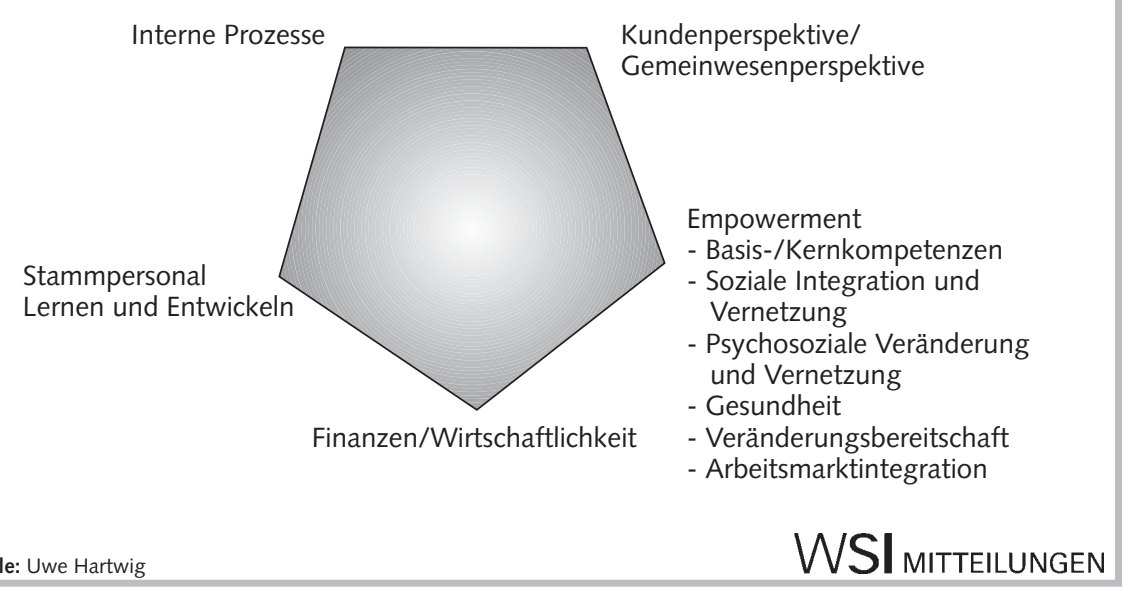

Qualifizierung unter realen Produktionsbedingungen die Arbeitsfähigkeit nachhaltig erhöhen und bessere Integrationschancen bieten soll (Werkstatt Frankfurt 2006, 2007).

\subsection{SOCIAL BALANCED SCORECARD}

Die als Begleitforschung7 konzipierte Untersuchung setzte sich zum Ziel, das bestehende Kennzahlensystem zur Erfolgsermittlung qualifizierender Beschäftigung auf der Basis einer noch weiter zu entwickelnden Social Balanced Scorecard zu evaluieren und zu optimieren. Sie erweitert die Balanced Scorecard (Kaplan/ Norton 1997), mit der „pragmatische Wege zur Ableitung von validen Aussagen über die Gesamteffizienz einer Organisation " (Becker/ Moses 2004, S. 44) gefunden werden sollen. ${ }^{8}$ Effizienz wird betriebswirtschaftlich dabei als ein Indikator der Wertschöpfung gesehen. Die Balanced Scorecard soll der Unternehmensführung und den einzelnen Beteiligten ermöglichen, die Vision eines ganzheitlich ausgerichteten Unternehmens in einem umsetzungsorientierten Instrument auf gezielte Aktivitäten zu übertragen. Über die Kommunikation zu den verschiedenen Ebenen des Unternehmens können bereichsspezifische und Unternehmensziele auf diese Strategie abgestimmt und damit das Verständnis der Beteiligten für die langfristige Perspektive und deren Identifikation mit den formulierten Zielen erhöht werden. Die dafür benötigten Ressourcen und Erfolgskomponenten sind regelmäßig zu hinterfragen und bei Bedarf zu modifizieren. Das heißt, dass die eingeleiteten Maßnahmen zur Zielerreichung, die unterstellten Ursache-Wirkungs-Beziehungen sowie die strategischen Ziele tur- nusmäßig im Hinblick auf die identifizierten Indikatoren, die definierten Kennzahlen und deren Größe überprüft werden. Die Balanced Scorecard stellt kein isoliertes Kennzahleninstrument, sondern eine Verbindung zwischen Vision bzw. langfristigen Zielen und deren Umsetzung dar.

$\mathrm{Zu}$ jeder Perspektive gilt es, signifikante Indikatoren zu identifizieren und diese zu einem Gesamtsystem zusammenzufassen. Durch die Offenheit des Systems ist eine Erweiterung auf zusätzliche Perspektiven möglich. Dies ist der zentrale Ansatzpunkt, um das Instrument für die Sozialwirtschaft nutzbringend zu erweitern. Für Qualifizierungs- und Beschäftigungsunternehmen wird die traditionelle Balanced Scorecard dabei um die Gemeinwesenperspektive und eine teilnehmerinnen- und teilnehmerbezogene soziale Komponente (Empowerment) zur Social Balanced Scorecard erweitert (Abbildung 1).

\footnotetext{
Gegenüber einem durchaus denkbaren Ansatz des Experiments zur Untersuchung der potenziellen Erhöhung der Beschäftigungswahrscheinlichkeit, wie es Eichhorst/Zimmermann (2007) mit einem Kontrollgruppenvergleich vorschlagen, schien uns der formative Ansatz mit der Befragung derselben Kohorte zu zwei Zeitpunkten angemessener, um qualitative und prozessuale Effekte, die ihre eigene Zeit der Entfaltung benötigen, zu untersuchen.

8 Die Balanced Scorecard (wortgemäß "ausgewogene Wertungs-/Punkteliste") geht auf Robert S. Kaplan und David P. Norton zurück. Diese hatten zu Beginn der 1990er Jahre die Balanced Scorecard als ein offenes Steuerungsinstrument entwickelt, das, ausgehend von der unternehmerischen $\mathrm{Vi-}$ sion, durch die Einbeziehung mehrerer Wirkungsebenen eine ganzheitliche Sichtweise ermöglicht und z. B. auch soziale Aspekte zur gezielten Steuerung des Unternehmensergebnisses einbeziehen kann.
} 


\begin{tabular}{|ll|}
\hline Übersicht 1: Indikatoren für Basis- & und Kernkompetenzen \\
\hline Lernkompetenz & $\begin{array}{l}\text { Lernbereitschaft, Aufnahmebereitschaft, } \\
\text { Lernfähigkeit, Qualifizierungsbereitschaft, } \\
\text { Selbstlernkompetenz }\end{array}$ \\
\hline Arbeitstugenden & $\begin{array}{l}\text { Zuverlässigkeit, Pünktlichkeit, } \\
\text { Verantwortungsbewusstsein, Pflichtgefühl, } \\
\text { Einsatzbereitschaft, Fleiß, Sorgfalt, Umgang mit } \\
\text { Arbeitsmitteln, Ordnung am Arbeitsplatz }\end{array}$ \\
\hline Organisationskompetenz & $\begin{array}{l}\text { Systematisches Arbeiten, selbstständiges } \\
\text { Arbeiten, Selbstmanagement, Arbeitsorganisation }\end{array}$ \\
\hline Sozialkommunikative Kompetenz & $\begin{array}{l}\text { Team- und Konfliktfähigkeit, Kooperationsfähigkeit, } \\
\text { Kommunikationsfähigkeit, interkulturelle } \\
\text { Kompetenz, Verhalten, Auftritt }\end{array}$ \\
\hline Sprachkompetenz & Sprachvermögen, Ausdrucksvermögen, Sprachen \\
\hline Leistungskompetenz & $\begin{array}{l}\text { Belastbarkeit, Ausdauer, Leistungsfähigkeit, } \\
\text { Arbeitsleistung }\end{array}$ \\
\hline Fachkompetenz & Fachwissen, Fachübergreifendes Wissen \\
\hline Quelle: Zusammenstellung der Autoren. & \\
\hline
\end{tabular}

Die Empowerment-Komponente umfasst mit den Zielgrößen Basis-/Kernkompetenzen, soziale Integration, Gesundheit, Veränderungsbereitschaft und Arbeitsmarktintegration ein erweitertes Konzept dessen, was Menschen erst in die Lage versetzt, ein individuelles Selbstmanagement zu entwickeln, und fördert so die Integration auch jenseits des ersten Arbeitsmarktes. Zugleich sind dies notwendige Voraussetzungen für reale Arbeitsmarktchancen. Das Ziel der gesellschaftlichen Integration beinhaltet also die Entfaltungsmöglichkeit subjektiver Fähigkeiten und Potenziale, eine gleichere Verteilung von Lebenschancen und die Bereitstellung individueller Qualifizierungsmöglichkeiten.

Unser Kompetenzbegriff meint auch die Befähigung, mit neuen Situationen und bisher unbekannten Handlungsanforderungen erfolgreich umgehen zu können (Erpenbeck 2003; Koch/Kupka 2007, S. 27). Als dynamischer Kompetenzbegriff geht er von den entwicklungsfähigen Fähigkeiten des Einzelnen aus (Lang-von Wins/Triebel 2006) und versteht das Erlernen neuer Kompetenzen als komplexes Handlungsspiel von Wissen, Überzeugungen und Handlungstendenzen. Des Weiteren gehen wir davon aus, dass Kompetenzen gemessen werden können.

\subsection{DIE ERHEBUNG}

Die Empowerment-Komponente der Social Balanced Scorecard wurde mit einem Methodenmix quantitativer und qualitativer Verfahren der Sozialforschung erfasst. Dabei kamen, aufbauend auf größtenteils standardisiert vorliegenden Fragen, teilnehmende Beobachtungen und leitfadengestützte Interviews zum Einsatz.
Künftig soll ein Vorher-Nachher-Vergleich zu zwei Zeitpunkten das Verfahren abrunden.

Im Fragebogeninterview schätzen die Arbeitslosen selbst ihre personalen Kompetenzen zu Beginn und Ende der Qualifizierungsmaßnahmen (in der Regel nach acht bis zwölf Monaten) ein. So kann erhoben werden, ob sich die Kompetenzen im Verlauf der Maßnahme in dieser Hinsicht weiterentwickelt haben und sich entsprechend auch die Beschäftigungsfähigkeit erhöht hat. Im Zeitverlauf ergeben sich damit Kennzahlen sozialer Arbeit, die über die Feststellung einer (nachhaltigen) Integrationsquote hinausgehen und differenziert die Wirkungsweise einzelner Maßnahmen in Bezug auf Kompetenzsteigerung und Empowerment-Prozesse aufzeigen.

Die Größenordnung der bereits durchgeführten teilnehmenden Beobachtung belief sich auf 15 Beobachtungseinheiten mit insgesamt 80 Teilnehmenden der Hamburger Arbeit $(\mathrm{HAB})(\mathrm{n}=80, \mathrm{~N}=303)$ sowie drei Beobachtungseinheiten mit zehn Teilnehmenden aus den Betriebsstätten der Werkstatt Frankfurt ( $\mathrm{n}=10, \mathrm{~N}=700)$. Explorative leitfadengestützte Interviews wurden am Standort Hamburg mit knapp $5 \%$ der Erwerbslosen der HAB $(\mathrm{n}=14$ : davon 5 Frauen, 9 Männer, 9 Deutsche, 5 nicht Deutsche im Alter von 30 bis 53 Jahren) in der sogenannten Vorbereitungsphase und mit einer Fallmanagerin geführt.

$\mathrm{Zu}$ Projektbeginn lag den Sozialunternehmen ein Indikatorenkatalog als Basis eines Fragebogens vor (Übersicht 1). Er gründet auf dem Kompetenzansatz von Heyse/Erpenbeck (2004) und wurde in einer interdisziplinären Arbeitsgruppe von So- zialwissenschaftlern und Praktikern überarbeitet, ergänzt und hinsichtlich Aussagekraft, Validität, Definition und Praktikabilität geprüft. Indikatoren für Kompetenzen und den Erfolg von Empowerment wurden definiert und in Form von Kennzahlengruppen in Basis-/Kernkompetenzen, Soziale Integration und Vernetzung, Psychosoziale Veränderung und Gesundheit, Veränderungsbereitschaft und Arbeitsmarktintegration geclustert (Abbildung 1).

Neben Basisdaten zur Soziostruktur wurden die zu Projektbeginn vorliegenden Indikatoren für die Beurteilung von Basis- und Kernkompetenzen modifiziert: Selbstständigkeit wurde an Eigeninitiative, Eigenverantwortung, Selbstbewusstsein und Motivation festgemacht. Weitere Indikatoren zu Soft Skills sowie zur psychosozialen Veränderung und Gesundheit ergänzten den Katalog. Die Veränderungsbereitschaft wurde bezüglich der räumlichen, zeitlichen, materiellen und tätigkeitsbezogenen Kategorien abgefragt, bevor mit der Nachhaltigkeit der Maßnahme Indikatoren zur Arbeitsmarktintegration gewonnen wurden. Die Gemeinwesen- und Teilhabeperspektive wurde einbezogen, indem nach möglichen Betätigungen oder Wünschen ehrenamtlicher und anderer Freizeitaktivitäten im privaten und öffentlichen Umfeld sowie Beziehungsnetzwerken, Zeit- und Alltagsstrukturierung gefragt wurde. Auf einer vierfachen Antwortskala sollten sodann in Form von Zustimmungssätzen Einschätzungen abgegeben werden.

Für die Erstellung des Leitfadens wurden Dimensionen des subjektiven Arbeitshandelns (zeitlich, sozial, ökonomisch, räumlich sowie Grad der Selbstrationalisierung, Selbstökonomisierung und Selbstkontrolle; vgl. Lohr 2003; Pongratz/Voß 2000) in eine systematische Reihenfolge gebracht und den Empowerment-Indikatoren zugeordnet. Die Themen der eingesetzten Fragen orientierten sich an der Erwerbsbiografie von Langzeitarbeitslosen. So wurde unter dem Begriff der „Arbeitsmarkthistorie“ zunächst erfragt, wie lange der Teilnehmer beschäftigt war, wie viele Beschäftigungsmaßnahmen absolviert wurden und wie die Vorbereitungsphase beurteilt wird.

Da auch Erwerbslosen zunehmend Anforderungen einer selbstorganisierten und 
marktförmigen Lebensführung ${ }^{9}$ abverlangt werden, wurden die Indikatoren hinsichtlich der erkenntnisleitenden Frage nach den spezifischen subjektiven Bewältigungsformen von Arbeitslosigkeit um die Punkte Alltagsorganisation, Arbeits- und Lebenseinstellung sowie Haltungstyp erweitert (vgl. Luedtke 1998).

\section{Ergebnisse der ersten Evaluationsphase}

\subsection{BEFUNDE}

Für die arbeitsmarktpolitische Praxis wurde bei der Auswertung deutlich, dass die tradierten Bewertungskriterien der gängigen Qualifizierungsansätze (Aufbau sekundärer Arbeitstugenden/persönlicher und sozialer Basiskompetenzen wie z. B. Fleiß, Pünktlichkeit, Pflichtgefühl, Sorgfalt und Ordnung) nicht nur an der Zielsetzung vorbeizugehen scheinen, die Kompetenzen Langzeitarbeitsloser im Hinblick auf Beschäftigungsfähigkeit/Employability zu erhöhen bzw. zu bewerten. Vielmehr stützen die vorläufigen Ergebnisse die jüngsten Aussagen zur "autoritär-aktivierend(en)“ (Oschmiansky et al 2007, S. 291) Arbeitsmarktpolitik. Zudem evozieren Fragen nach offensichtlich sozial erwünschten Verhaltensweisen (wie etwa Pünktlichkeit) verzerrte Antworten. Die hier nur exemplarisch zu verstehenden Ausführungen der Befragten verweisen auf heterogene Befunde: Allen Befragten ist gemeinsam, dass sie ihre Arbeitslosigkeit zunächst als einen massiven Bruch und zuweilen auch als sozialen Abstieg wahrnehmen. Unterschiedliche Gründe, wie befristete, ausgelaufene Verträge, Firmenkonkurs, familiäre Belastungen und Gesundheitsprobleme, lösten die Arbeitslosigkeit aus. Alle Betroffenen sind eindeutig mit dem Wunsch nach einem „festen Job“ auf den Arbeitsmarkt hin orientiert. Die angebotenen AGHs werden hingegen unterschiedlich beurteilt und zum einen als Hilfe zur regelmäßigen Lebensführung, beruflichen Orientierung und Zeitstrukturierung sowie Teilhabemöglichkeit erkannt. Zum anderen werden manche Angebote als stumpfsinnig und gängelnd empfunden. Die erhöhte Anforderung, sich viele Informationen selbst beschaffen zu müssen, wird beklagt. Von den Befragten werden darüber hinaus die Merk- male "Organisation des Arbeitslebens", "Arbeits- und Lebenseinstellung" sowie „Haltungstyp" mehrheitlich positiv erfüllt. Bei vier bis fünf Befragten (ca. ein Drittel) ist eine eher negative, passive bis hin zu einer destruktiven Haltung zu erkennen. Betrachtet man diese Merkmale gemeinsam mit dem Merkmal „Organisation des Alltags“, welches nur partiell als gelingend angesehen werden kann, ist zu beobachten, dass bei den Teilnehmern eher geringe Handlungsressourcen vorhanden sind. Bei der Dimension „Selbst-Ökonomisierung“ im Sinne einer marktorientierten Haltung kann dagegen bei 30-50 \% der Befragten von einem hohen Selbstvermarktungsund Aktivierungsdruck gesprochen werden. Hinsichtlich der alltäglichen Lebensführung lässt sich feststellen, dass die zugeordneten Merkmale bei acht von 14 Langzeitarbeitslosen zu knapp über $50 \%$ erfüllt werden und damit auch hier eine partielle Selbstrationalisierung der Erwerbslosen vorliegt.

Daraus kann nach dem bisherigen Zwischenstand als Empfehlung abgeleitet werden, dass die eigentliche Stoßrichtung angebotener Maßnahmen sich entsprechend komplexerer und realistischerer Anforderungen der Zielgruppen (z. B. Teamfähigkeit, Selbstorganisations- und (Fremd-) Sprachvermögen wie auch Selbstrationalisierung) auf die spezifische Steigerung der gesellschaftlichen Teilhabechancen und damit der Employability abzielen sollte.

Die Frage, aus welchen Gründen dieses Potenzial von den Teilnehmenden nicht auf dem ersten Arbeitsmarkt genutzt werden kann, verweist darauf, dass neben den zweifelsohne relevanten gesundheitlichen Beeinträchtigungen vor allem spezifische fachliche und strukturelle Hemmnisse und weniger individuelle, selbst verschuldete Verhaltensdefizite bestehen, die der Erreichung des ersten und regional unterschiedlich intakten Arbeitsmarktes entgegenstehen.

Der starke Wunsch aller Befragten nach einer Stelle ist in diesem Kontext gleichwohl überzeugend zu vernehmen und verweist auf die Relevanz von Arbeit als Grundlage von Identität und Teilhabemöglichkeiten, die in und von Sozialunternehmen auch jenseits des ersten Arbeitsmarktes geschaffen werden können. Koch und Kupkas Erwägung bleibt dabei weiter zu diskutieren:

„Wenn es wirklich stimmt, dass ein großer Teil von erwerbsfähigen Hilfebedürftigen wegen geringer Produktivität keine Chance auf eine reguläre Beschäftigung hätte und einer Art von beschützter Beschäftigung bedarf, muss man sich fragen, ob nicht doch für einen Teil dieses Personenkreises andere Sozialsysteme angemessener wären" (Koch/Kupka 2007, S. 32).

\subsection{FAZIT UND AUSBLICK}

Wenngleich die Maßnahmen der untersuchten Sozialunternehmen ein nahezu uneinlösbares Integrationsversprechen und Dilemma bergen, weil sie durch die Kriterien der Zusätzlichkeit, des öffentlichen Interesses und der Marktferne stark eingeschränkt sind, können mit den Qualifizierungsangeboten Prozesse des Empowerments und der sozialen Teilhabe in Gang gesetzt werden. Social Scoring ermöglicht hier nicht nur, Daten zur Sozialstruktur, zu Basis- und Kernkompetenzen, Integration und psychosozialen Veränderung jenseits diskriminierender „Creaming“-Effekte zu erfassen. Vielmehr können besser als bei statischen Momentaufnahmen, dies zeigen die ersten Zwischenergebnisse, dynamische Prozesse der Kompetenzgewinnung eingefangen werden. Damit wird neben den Aspekten der Struktur- und Ergebnisqualität von Qualifizierungsangeboten vermehrt die Prozessqualität und Wirkungsmessung relevant (vgl. Merchel 2001; Gerull 1999). Allerdings geht aus den ersten Befunden auch hervor, dass die arbeitsmarktpolitischen Maßnahmen bisher nicht ausreichend auf die wachsenden Selbstorganisations- und Lebensführungszwänge des ersten Arbeitsmarkts vorbereiten, die von leistungsgeminderten und mehrfach eingeschränkten Langzeitarbeitslosen selbstständig und eigenverantwortlich kaum zu erfüllen sind.

In einem nächsten Schritt wird es darum gehen, einen indikatorengestützten

\footnotetext{
Das zugrunde gelegte theoretische Konzept der subjektorientierten Arbeits- und Berufssoziologie fragt dabei auf einer übergeordneten Ebene auch nach der Lebensführung Betroffener, die die Belastungen durch Arbeitslosigkeit in einem äußerst heterogenen Feld der Verarbeitungs- und Haltungsmuster spezifisch und abhängig von den zur Verfügung stehenden Ressourcen und Kompetenzen zu bewältigen haben (vgl. Luedtke 1998). Vor dem Hintergrund der Lebensführungszwänge Arbeitsloser bedeutet dies auch, dass im Laufe längerer Phasen von Arbeitslosigkeit die Anerkennung und auch das (Selbst-)Vertrauen in diese personalen Kompetenzen schwinden können.
} 
Fragebogen mit ca. 80-100 Teilnehmenden an den Standorten Frankfurt und Hamburg zu zwei Zeitpunkten einzusetzen. $\mathrm{Ob}$ und wie sich nach Ende der Maßnahme mit einem größeren zeitlichen Abstand die Arbeitsgelegenheiten bewährt haben werden, kann zum gegenwärtigen Zeitpunkt noch nicht beantwortet werden. Sicherlich wäre es sinnvoll, hier eine weitere Befragung sowie einen Kontrollgruppenvergleich anzuschließen.

\section{ITERATUR}

Bartelheimer, P. (2004): Teilhabe - Gefährdung und Ausgrenzung als Leitbegriffe der Sozialberichterstattung, in: SOFI-Mitteilungen 32, S. 47-61

Baethge-Kinsky, V. (2006): Was bringt die Wirkungsforschung? Wie kann und muss man sie nutzen?, www.monitor-arbeitsmarktpolitik.de (Download 24. Januar 2007), S. 1-15

Baethge-Kinsky, V. (2007): Transformation statt Erosion arbeitsmarktpolitischer Qualifizierung - Lehren aus der Evaluation der Hartz-Gesetze, in: WSI-Mitteilungen 6, S. 312-319

Becker, W./Moses, H. (2004): Controlling in karitativen Nonprofit-Organisationen, Bamberger Betriebswirtschaftliche Beiträge, Bd. 133, Bamberg Bundesagentur für Arbeit (BA) (2007): Arbeitsmarktstatistik, Januar, Nürnberg

Castel, R. (2000): Die Metamorphosen der sozialen Frage - Eine Chronik der Lohnarbeit, Konstanz

Compass/IMU Institut/ PIW/ SÖSTRA (2006): Evaluation der Maßnahmen zur Umsetzung der Vorschläge der Hartz-Kommission.

Arbeitspaket 1: Wirksamkeit der Instrumente. Modul 1c: Arbeitsbeschaffungsmaßnahmen. Endbericht, Berlin/Bremen/Hamburg

Deeke, A./Kruppe, T. (2003): Beschäftigungsfähigkeit als Evaluationsmaßstab? Inhaltliche und methodische Aspekte der Wirkungsanalyse beruflicher Weiterbildung im Rahmen des ESF-BA-Programms, in: IAB Werkstattbericht. Diskussionsbeiträge des Instituts für Arbeitsmarkt- und Berufsforschung der Bundesanstalt für Arbeit 1, vom 10.2.

Eichhorst, W./Zimmermann, K. F. (2007): Dann waren's nur noch vier... Wie viele (und welche) Maßnahmen der aktiven Arbeitmarktpolitik brauchen wir noch? Eine Bilanz nach der Evaluation der Hartz-Reformen, in: IZA Discussion Paper 2605, Februar, S. 1-33

Erpenbeck, J./v. Rosenstiel L. (2003): Einführung, in: Dies. (Hrsg.): Handbuch Kompetenzmessung, Stuttgart, S. IX-XL

Gerull, P. (1999): Selbstbewertung des Qualitätsmanagements. Eine Arbeitshilfe, Bundesministerium für Familie, Senioren, Frauen und Jugend, Berlin

Hamburger Arbeit Beschäftigungsgesellschaft mbH (HAB) (Hrsg.)

(2005): Geschäftsberichte 1999-2005, Hamburg

Herriger, N. (1997): Empowerment in der Sozialen Arbeit. Eine Einführung, Stuttgart.

Heyse, V./Erpenbeck, J. (2004): Kompetenztraining. 64 Informationsund Trainingsprogramme, Stuttgart

Kaplan, R. S./Norton, D. P. (1997): Balanced Scorecard - Strategien erfolgreich umsetzen, Stuttgart

Kettner, A. /Rebien, M. (2007): Soziale Arbeitsgelegenheiten, Einsatz und Wirkungsweise aus betrieblicher und arbeitsmarktpolitischer Perspektive. IAB-Forschungsbericht 2, Nürnberg

Koch, S./Kupka, P. (2007): Geförderte Beschäftigung für leistungsgeminderte Langzeitarbeitslose? Expertise im Auftrag der Friedrich-Ebert-Stiftung, Oktober, Bonn, htttp://www.fes.de/wiso
Kreft, D./Mielenz, I. (2005): Wörterbuch Soziale Arbeit, Aufgaben, Praxisfelder, Begriffe und Methoden der Sozialarbeit und Sozialpädagogik, Weinheim und München

Kronauer, M. (2001): Einleitung, in: Häußermann, H./Kronauer, M./ Siebel, W. (Hrsg.): Stadt am Rand, Frankfurt/Main, S. 7-12

Lang-von Wins, T./Triebel, C. (2006): Kompetenzorientierte Laufbahnberatung, Heidelberg

Ludwig-Mayerhofer, W. (2005): Arbeitslosigkeit, in: Abraham, M./Hinz, T. (Hrsg.): Arbeitsmarktsoziologie. Probleme, Theorien, empirische Befunde, Wiesbaden, S. 199-239

Luedtke, J. (1998): Lebensführung in der Arbeitslosigkeit. Differentielle Problemlagen und Bewältigungsmuster, Pfaffenweiler

Lohr, K. (2003): Subjektivierung von Arbeit. Ausgangspunkt einer Neuorientierung der Industrie- und Arbeitssoziologie? in: Berliner Journal für Soziologie 4, S. 511-529

Merchel, J. (2001): Qualitätsmanagement in der sozialen Arbeit. Ein Lehrund Arbeitsbuch, Münster

Mertens, D. (1974): Schlüsselqualifikationen: Thesen zur Schulung für eine moderne Gesellschaft, in: Mitteilungen aus der Arbeitsmarkt- und Berufsforschung 1, S. 36-43

Oschmiansky, F./Mauer, A./Schulze Buschoff, K. (2007): Arbeitsmarktreformen in Deutschland - Zwischen Pfadabhängigkeit und Paradigmenwechsel, in: WSI Mitteilungen 6, S. 291-297

Osenberg, G. (1995): Beschäftigungsgesellschaften - Ziele, Konzepte und Eignung, Europäische Hochschulschriften: Reihe 5, Volks- und Betriebswirtschaft Bd. 1720, Frankfurt/Main

Penz, R. (2007): Wirkungsforschung, arbeitsmarktpolitische Ziele und politisches Lernen. Einige Anmerkungen zum Beitrag von Volker BaethgeKinsky, in: www.monitor-arbeitsmarktpolitik.de (Download 24. Januar 2007)

Pongratz, H./Voß, G. G. (2000): Vom Arbeitnehmer zum Arbeitskraftunternehmer. Zur Entgrenzung der Ware Arbeitskraft, in: Minssen, $\mathrm{H}$. (Hrsg.): Begrenzte Entgrenzungen. Wandlungen von Organisation und Arbeit, Berlin, S. 225-247

Renn, H./Schoch, D. (2005): Grundsicherung für Arbeitsuchende (SGBII). Das neue Sozialleistungsrecht für hilfebedürftige erwerbsfähige Personen, Baden-Baden

Sozialgesetzbuch II (2003): Zweites Buch (II) - Grundsicherung für Arbeitsuchende, Berlin

Sozialgesetzbuch III (1997): Drittes Buch (III) - Arbeitsförderung, Berlin Tractenberg, L./Streumer, J./van Zolingen, S. (2002): Career counselling in the emerging post-industrial society. International journal for educational and vocational guidance 2, S. 85-99

Werkstatt Frankfurt (Hrsg.) (2007): Das Unternehmen Werkstatt Frankfurt, Frankfurt/Main 